\title{
Prognostic value of C-reactive protein/albumin ratio in predicting overall survival of Chinese cervical cancer patients overall survival: comparison among various inflammation based factors
}

\author{
Xia He*, Jian-Pei Li, Xiao-Hua Liu, Jing-Ping Zhang, Qiu-Yao Zeng, Hao Chen ${ }^{\varpi}$ and Shu-Lin Chen ${ }^{凶}$ \\ State Key Laboratory of Oncology in South China, Collaborative Innovation Center for Cancer Medicine, Sun Yat-sen University Cancer Center, Guangzhou \\ 510060, China. \\ *These authors contributed equally to this work. \\ $\triangle$ Corresponding authors: chenhao@sysucc.org.cn (H.C.); Tel./Fax: +86-20-8734-3268 and chenshl@sysucc.org.cn (S.-L.C.); Tel./Fax: +86-20-8734-3438 \\ (C) Ivyspring International Publisher. This is an open access article distributed under the terms of the Creative Commons Attribution (CC BY-NC) license \\ (https://creativecommons.org/licenses/by-nc/4.0/). See http://ivyspring.com/terms for full terms and conditions.
}

Received: 2017.10.14; Accepted: 2018.01.24; Published: 2018.04.27

\begin{abstract}
Background: Many studies have shown the prognostic value of inflammation based factors in different cancers. This work aimed to explore the prognostic value of pretreatment C-reactive protein/albumin (CRP/Alb) ratio in patients with cervical cancer, and compared to other inflammatory prognostic factors, such as neutrophil/lymphocyte ratio(NLR), Glasgow prognostic score (mGPS), prognostic index (PI), platelet/lymphocyte ratio (PLR), prognostic nutritional index (PNI), clinicopathological parameter and squamous cell carcinoma antigen (SCC-Ag).

Methods: This study was a retrospective analysis of the data related to 229 patients with newly diagnosed cervical cancer. The potential prognostic factors were evaluated by univariate and multivariate survival analysis. The correlation between CRP/Alb ratio and other prognostic factors were analyzed by Chi-Square or Fisher's exact test.

Results: Multivariate analyses showed that CRP/Alb ratio was an independent predictor of overall survival (OS) in cervical squamous cell carcinoma $(\mathrm{SCC})(\mathrm{HR}$, hazard ratio $=2.529 ; p=0.045)$, but not in all cases of cervical cancer. However, NLR was a prognostic factor in the whole cervical cancer $(\mathrm{HR}=$ 2.47; $p=0.020)$ as well as in SCC subgroup $(H R=2.28 ; p=0.038)$. Spearman's rank correlation analysis revealed that NLR showed a positive correlation with CRP/Alb ratio $(p<0.001)$. The combined index of NLR and CRP/Alb ratio could enhance the prognostic value compared to NLR or CRP/Alb ratio alone. Moreover, a high CRP/Alb ratio $>0.022$ was associated with older patients $(p<0.001)$ and more advanced International Federation of Gynecology and Obstetrics (FIGO) stages $(p<0.001)$. In addition, NLR and CRP/Alb ratio were associated with SCC-Ag concentration in SCC. Furthermore, CRP/Alb ratio was a superior prognosis factor than $\mathrm{MGPS}, \mathrm{PI}, \mathrm{PLR}$ and $\mathrm{PNI}$ in SCC. Moreover, positive correlation was present among SCC-Ag, NLR and CRP/Alb ratio.

Conclusions: CRP/Alb ratio might be considered as a novel prognosis factor and combined with NLR could improve the accuracy of OS prediction in patients with cervical cancer as well as its most common histological SCC subtypes.

Key words: Cervical cancer, inflammation based factors, C-reactive protein/albumin ratio, neutrophil/lymphocyte ratio(NLR), overall survival
\end{abstract}

\section{Introduction}

Cervical cancer is the second most commonly diagnosed cancer and the third leading cause of cancer death among females in less developed countries [1]. Nearly one-third of patients with 
cervical cancer die due to disease recurrence or progression [2]. In order to take a precise individual treatment in patients with cervical cancer, the discovery of suitable markers for a correct prognosis evaluation is urgently needed.

Currently, clinical stages and several pathological features, including invasion depth, lymph node (LN) status and tumor size are often used to predict recurrence in cervical cancer [3-4]. However, some researches showed that clinical stages were not accurate enough [5-6] and the pathological risk factors for prognosis cannot be used in patients without surgery. Furthermore, few markers can accurately predict the prognosis and their validity is still controversial.

Squamous cell carcinoma (SCC) accounting for about $85 \%$ is the most common histological subtypes of cervical carcinoma [7]. Squamous cell carcinoma antigen (SCC-Ag) is the most commonly used biomarker to screen SCC of the cervix; it is related to tumor stage, tumor size, the amount of residual tumor after treatment and recurrence [8]. SCC-Ag in high levels can precede the clinical diagnosis of recurrent disease in $46 \%-92 \%$ of the cases; however, its effectiveness in prognosis prediction is still under debated [9-10].

The systemic inflammatory response is associated with carcinogenesis, tumor progression and metastasis (Balkwill and Mantovani 2001; Candido and Hagemann 2013; Diakos et al. 2014; Werb 2002). There are substantial evidences that the host systemic inflammatory response is an important independent outcomes predictor in cancer patients and that pretreatment measures of the systemic inflammatory parameters can be used to independently predict cancer survival. C-reactive protein albumin (CRP) test is great significance in the early diagnosis, differential diagnosis and observation of curative effects. Suyang Guo et al. aslo confirmed the role of CRP in the early diagnosis of cervical cancer recurrence [11]. Several nutrition-based and (or) inflammatory factors have been applied to predict the survival time in many cancers, such as neutrophil/lymphocyte ratio (NLR) [12], platelet/ lymphocyte ratio (PLR) [16], C-reactive protein albumin (CRP/Alb) ratio [13], modified Glasgow prognostic score (mGPS) [14], prognostic index (PI) [12] and prognostic nutritional index (PNI) [15]. Among these biomarkers, white cell counts and mGPS were previously used as inflammatory parameters to predict survival of cervical cancer patients, and demonstrated that NLR and mGPS were related to survival prognosis in patients with cervical cancer.

The CRP/Alb ratio as a novel prognostic markers has been reported in hepatocellular carcinoma, small cell lung carcinoma and esophageal squamous cell carcinoma. However, it is unknown whether pretreatment $\mathrm{CRP} / \mathrm{Alb}$ ratio is a prognostic marker in patients with cervical cancer, and whether its prognostic value is or not superior to other inflammatory prognostic factors. Therefore, the aim of our study was to investigate the prognostic value of CRP/ Alb ratio in patients with cervical cancer, and compared it to other prognostic factors (CRP, PNI, PI and mGPS), cellular parameters (NLR, PLR) and SCC-Ag.

\section{Materials and Methods}

\section{Ethics statement}

All patients provided written informed consent to participate to this survey. The study was approved by the Ethics Committee of Sun Yat-Sen University Cancer Center.

\section{Study population}

Patients with first diagnosed cervical cancer who were treated at Sun Yat-sen University Cancer Center from September 2007 to March 2009 were retrospectively enrolled in our study. All the data were collected before treatment. The exclusion criteria were the following: 1 . the patients without pathological diagnosis or with previous or concomitant malignancies; 2 . incomplete records in the database of medical information; 3. patients lost during follow-up, or patients who died because of non-cancer causes; 4 . in order to eliminate the influences of non-cancer diseases on inflammation-based prognostic factors, we excluded patients with rheumatoid diseases and acute infection. Finally, 229 patients were enrolled in our study. The authenticity of this article has been validated by uploading the key raw data onto the Research Data Deposit public platform (www. researchdata.org.cn), with the approval RDD number as RDDA2018000550.

\section{Clinicopathological features}

Baseline participants' characteristics of participants were gathered from medical records including age, FIGO stages, histological typing, histological grade, tumor size, and lymph node(LN) metastasis were collected from medical records. Patients with International Federation of Gynecology and Obstetrics (FIGO) 2009 stage I (IA 17, IB1 89, IB2 35), stage II (IIA 47, IIB 28), and stage III/IV (III 12, IV 1) cancer were included in this retrospective analysis. Overall survival (OS) was defined as the time from the date of the diagnosis until the date of death, or the date of the last follow-up. Patient follow-up was completed up to January 21, 2016. 


\section{Nutritional and inflammatory parameters}

All the samples were collected at the time of diagnosis before any treatment, including white cells, neutrophils, lymphocytes, platelets, level of serum CRP, albumin concentration and SCC-Ag level. The count of white blood cells, neutrophils, lymphocyte and platelets were collected by an automated hematology system (Sysmex XE-5000, Kobe, Japan). CRP and albumin were measured by an automatic biochemical analyzer (Hitachi 7600, Tokyo, Japan). SCC-Ag was measured using a chemiluminescence microparticle immunoassay (Abbott Architect i2000, USA). $\mathrm{CRP} / \mathrm{Alb}$ ratio was defined as the serum CRP level divided by the serum Alb level. NLR was defined as the neutrophil count divided by lymphocyte count. PLR was defined as the absolute platelet count divided by the absolute lymphocyte count. mGPS consists of serum CRP and albumin levels (mGPS 2: CRP $>10 \mathrm{mg} / \mathrm{L}$ and albumin <35 g/L; mGPS 1: CRP $>10 \mathrm{mg} / \mathrm{L}$ and albumin $>35 \mathrm{~g} / \mathrm{L}$; mGPS 0 : CRP $\leq 10$ $\mathrm{mg} / \mathrm{L})$. PI: 0 for CRP $10 \mathrm{mg} / \mathrm{L}$ or less and white cell count $11 \times 10^{9} / \mathrm{L}$ or less, 1 if one of the two markers was higher, 2 if both of them were higher. PNI was calculated by the formula Alb $(\mathrm{g} / \mathrm{L})+5 \times$ lymphocyte count $\times 10^{9}$ /L: 0 for PNI > 45; 1 for PNI $\leq 45$ [16].

\section{The optimal cutoff prognostic values for CRPIAlb ratio, NLR and PLR}

The best cut-off value was determined by minimum P-value approach [17]. The CRP/Alb ratio cutoff point for cervical cancer specific survival was 0.022 (40th percentile value) with the maximum $\chi^{2}$ log-rank value of $9.181(p=0.002)$, and all patients were divided into either high or low CRP/Alb ratio group. NLR cutoff 1.60 (33th percentile value) and PLR cutoff 149.27 (66th percentile value) were selected as optimal cutoff level for assessing OS ( $\chi^{2}=6.830, p=$ 0.009 , and $\chi^{2}=5.544, p=0.019$, respectively) to divide the patients into high or low risk subsets.

\section{Statistical Analysis}

Statistical analyses were using SPSS 19.0 statistical software package (SPSS Inc., Chicago, IL, USA). Variables with significant prognostic value on univariate analysis were selected for multivariable Cox regression analysis adopting the forward stepwise method. The survival curves were plotted by the Kaplan-Meier method and compared with log-rank test. Chi-Square or Fisher's exact test was used to compare the categorical variables. The association between NLR and CRP/Alb Ratio was analyzed by Spearman's rank correlation. Receiver operating characteristics (ROC) curves and the areas under the curve (AUC) were used to evaluate the discriminatory ability. All reported $\mathrm{p}$ values were two-sided, and a $p$ value less than 0.05 was considered statistically significant.

\section{Results}

\section{Patient Characteristics}

A total of 229 patients were enrolled in our study. The overall median age was 44 years (age range, 28 to 79 years), the median survival time was 83 months, and the actuarial 7-year survival rate was $79.0 \%$. More than half of the patients were diagnosed at early stages (IA to IIA $=188 / 229,82.1 \%$ ) and with negative LN metastasis $(184 / 229,80.3 \%)$. Among all, 115 (G1 and G2, $50.2 \%$ ) patients were classified into the well-differentiated carcinoma category and 114 (G3, 49.8\%) patients were classified into the poor undifferentiated carcinoma category. Most of them received radical surgery as first-line treatment $(203 / 229,88.6 \%)$, and 153 of them underwent chemotherapy and(or) radiotherapy after surgery. The baseline characteristics and parameters of the 229 patients are shown in Table 1.

\section{Univariate Survival Analysis of All Prognostic Parameters}

In order to investigate the prognostic value of $\mathrm{CRP} / \mathrm{Alb}$ ratio and other prognostic factors, we performed survival analysis in cervical cancer and SCC subgroup. The univariate analysis results (Table 1) showed that FIGO stages, tumor size, LN, treatments, CRP, CRP/Alb ratio, NLR, PLR, mGPS, PI and SCC-Ag were significantly associated with OS in all patients, but in SCC subgroup, tumor size and PLR were not clear.

\section{Multivariate Analysis of All Prognostic Parameters}

All prognostic factors with $p$ less than 0.05 in univariate analysis underwent multivariate analysis (Table 1). FIGO stages [HR $=2.36$ (95\% CI:1.02-5.47), $p$ $=0.001], \mathrm{LN}$ metastasis $[\mathrm{HR}=0.47$ (95\% CI: 0.26-0.85), $p=0.013]$, treatments [HR $=2.77$ (95\% CI: 1.56-4.91), $p$ $=0.001]$ and NLR [HR $=2.47$ (95\% CI: 1.15-5.31), $p=$ 0.020 ] were independently associated with all patients OS. Besides, LN metastasis [HR $=0.41(95 \% \mathrm{CI}$ : $0.22-0.78), p=0.008]$, treatments [HR $=2.72(95 \% \mathrm{CI}$ : $1.46-5.07), p=0.002], \mathrm{CRP} / \mathrm{Alb}$ ratio $[\mathrm{HR}=2.36(95 \%$ CI:1.02-5.47), $p=0.045]$ and NLR [HR $=2.28(95 \%$ CI: 1.05-4.97), $p=0.038$ ] were identified to be independent prognostic factors for OS in SCC.

Kaplan-Meier survival curves according to stratified by CRP/Alb ratio (Fig. 1 a and d) and NLR (Fig. $1 \mathrm{~b}$ and e) revealed that the presence of systemic inflammatory response as elevated NLR and $\mathrm{CRP} / \mathrm{Alb}$ ratio was unprofitable for survival of all and SCC patients. CRP/Alb ratio dichotomisation showed 
the greater survival difference than NLR (74\% high vs $89 \%$ low), with a 7 -year survival rate of $72 \%$ (CRP/ Alb-high) vs 90\% (CRP/Alb-low) in all patients. These results were echoed in the SCC subgroup with estimated cumulative 7-year survival rate of $71 \%$ (CRP/Alb-high) vs 91\% (CRP/Alb-low) and NLR (74\% high and $88 \%$ low).

\section{Prognostic Significance of the Combined Index CRPIAlb ratio and NLR}

Further analysis of the relation between $\mathrm{CRP} / \mathrm{Alb}$ ratio and NLR was performed using the Spearman's rank correlation analysis. The results showed that the CRP/Alb ratio was positively associated with NLR $(r=0.235, p<0.001)$ (Fig. 2).
Methods combining CRP/Alb ratio with NLR might improve patient stratification in relation to OS. Thus, we stratified patients into three groups: (a) NLR-low and CRP/Alb-low, (b) NLR-high or CRP/Alb -high and (c) CRP/Alb-high and NLR-high (Fig. $1 \mathrm{c}$ and f), corresponding to low, intermediate and high risk groups. The cumulative 7-year OS rate in the 229 patients was $97 \%, 84 \%$ and $68 \%$ respectively, and in SCC subgroup was $97 \%, 83 \%$ and $68 \%$ respectively. When adjusted for other prognostic factors in the multivariate analysis (Table 1), both the high and intermediate risk groups had significantly poor prognosis than the low risk group.

Table 1. Pretreatment inflammatory parameters for overall survival indentified by univariate and multivariate analyses

\begin{tabular}{|c|c|c|c|c|c|c|c|c|}
\hline \multirow{3}{*}{ Characteristic } & \multicolumn{4}{|c|}{ All patients $(n=229)$} & \multicolumn{4}{|c|}{ SCC group $(n=198)$} \\
\hline & \multicolumn{2}{|c|}{ Univariate analysis } & \multicolumn{2}{|c|}{ Multivariate analysis } & \multicolumn{2}{|c|}{ Univariate analysis } & \multicolumn{2}{|c|}{ Multivariate analysis } \\
\hline & HR (95 \% CI) & $p$ value & HR (95 \% CI) & $p$ value & HR (95 \% CI) & $p$ value & HR (95 \% CI) & $p$ value \\
\hline \multicolumn{9}{|l|}{ Age(years) } \\
\hline$<44 / \geq 44$ & $1.50(0.82-2.74)$ & 0.185 & & & $1.65(0.86-3.17)$ & 0.135 & & \\
\hline \multicolumn{9}{|l|}{ FIGO stages } \\
\hline I/II/IIIand IV & $3.26(2.20-4.81)$ & $<0.001^{*}$ & $2.13(1.38-3.27)$ & $0.001^{*}$ & $2.81(1.84-4.29)$ & $<0.001^{*}$ & $1.58(0.99-2.55)$ & 0.058 \\
\hline \multicolumn{9}{|l|}{ Histologic typing } \\
\hline Sqc/ Adc/ Asc & $0.80(0.38-1.67)$ & 0.551 & & & & & & \\
\hline \multicolumn{9}{|l|}{ Histologic grade } \\
\hline G1 and G2/ G3 & $1.44(0.81-2.56)$ & 0.210 & & & $1.55(0.83-2.89)$ & 0.170 & & \\
\hline \multicolumn{9}{|l|}{ Tumor size, $\mathrm{cm}$} \\
\hline$<4 / \geq 4$ & $2.10(1.18-3.75)$ & $0.012^{*}$ & & & $1.80(0.96-3.38)$ & 0.069 & & \\
\hline \multicolumn{9}{|l|}{ LN metastasis } \\
\hline No/ Yes & $0.30(0.17-0.54)$ & $<0.001^{*}$ & $0.47(0.26-0.85)$ & $0.013^{*}$ & $0.30(0.16-0.55)$ & $<0.001^{*}$ & $0.41(0.22-0.79)$ & $0.008^{*}$ \\
\hline \multicolumn{9}{|l|}{ Treatments } \\
\hline $\begin{array}{l}\text { Sur / Sur and Che and(or) Rad/ Che and } \\
\text { (or) Rad }\end{array}$ & $4.28(2.55-7.18)$ & $<0.001^{*}$ & $2.77(1.56-4.91)$ & $0.001^{*}$ & $3.80(2.18-6.64)$ & $<0.001^{*}$ & $2.72(1.46-5.07)$ & $0.002^{*}$ \\
\hline \multicolumn{9}{|l|}{ NLR } \\
\hline$\leq 1.60 />1.60$ & $2.64(1.24-5.64)$ & $0.012^{*}$ & $2.47(1.15-5.31)$ & $0.020^{*}$ & $2.51(1.16-5.42)$ & $0.019^{*}$ & $2.28(1.05-4.97)$ & $0.038^{*}$ \\
\hline \multicolumn{9}{|l|}{ PLR } \\
\hline$\leq 149.27 />149.27$ & $1.95(1.10-3.43)$ & $0.021^{*}$ & & & $1.64(0.89-3.01)$ & 0.115 & & \\
\hline \multicolumn{9}{|l|}{ Neutrophil count $(\times 109 / \mathrm{L})$} \\
\hline$\leq 7.0 />7.0$ & $2.11(0.94-4.70)$ & 0.069 & & & $2.36(0.99-5.61)$ & 0.052 & & \\
\hline \multicolumn{9}{|l|}{ CRP(mg/L) } \\
\hline$\leq 10.0 />10.0$ & $3.02(1.41-6.46)$ & $0.004^{*}$ & & & $3.03(1.34-6.82)$ & $0.008^{*}$ & & \\
\hline \multicolumn{9}{|l|}{$\operatorname{Alb}(g / L)$} \\
\hline$\geq 35.0 /<35.0$ & $0.602(0.08-4.36)$ & 0.615 & & & & & & \\
\hline \multicolumn{9}{|l|}{ CRP/Alb } \\
\hline$\leq 0.022 />0.022$ & $3.18(1.54-6.57)$ & $0.002^{*}$ & & & $3.49(1.55-7.85)$ & $0.003^{*}$ & $2.36(1.02-5.47)$ & $0.045^{*}$ \\
\hline \multicolumn{9}{|l|}{ mGPS } \\
\hline $0 / 1 / 2$ & $3.02(1.41-6.46)$ & $0.004^{*}$ & & & $3.03(1.34-6.82)$ & $0.008^{*}$ & & \\
\hline \multicolumn{9}{|l|}{ PI } \\
\hline $0 / 1 / 2$ & 1.82(1.13-2.92) & $0.013^{*}$ & & & $1.95(1.16-3.27)$ & $0.011^{*}$ & & \\
\hline \multicolumn{9}{|l|}{ PNI } \\
\hline $0 / 1$ & $2.30(0.56-9.50)$ & 0.248 & & & $2.29(0.55-9.49)$ & 0.252 & & \\
\hline \multicolumn{9}{|l|}{ SCC-Ag } \\
\hline$\leq 1.5 />1.5$ & $2.40(1.35-4.27)$ & $0.003^{*}$ & & & $2.84(1.50-5.40)$ & $0.001^{*}$ & & \\
\hline \multicolumn{9}{|l|}{ NLR+CRP/Alb } \\
\hline $\begin{array}{l}\text { NLR-low+CRP/Alb-low / NLR-high or } \\
\text { CRP/Alb-high/NLR-high+CRP/Alb-high }\end{array}$ & $2.72(1.63-4.54)$ & $<0.001^{*}$ & $2.22(1.30-3.80)$ & $0.003^{*}$ & $2.63(1.54-4.48)$ & $<0.001^{*}$ & $2.32(1.32-4.07)$ & $0.003^{*}$ \\
\hline
\end{tabular}

*Statistically significant prognostic factor identified by univariate/multivariate analysis.

Abbreviation: FIGO: International Federation of Gynecology and Obstetrics, Sqc: Squamous carcinoma, Adc: Adenocarcinoma, Asc: Adenosquamous carcinoma, LN lymph node, Sur: Surgery, Che: chemotherapy, Rad: radiotherapy, NLR: neutrophil lymphocyte ratio, PLR: platelet lymphocyte ratio, CRP: C-reactive protein, WBC: white blood cell. CRP/Alb: C-reactive protein/Albumin ratio, mGPS: modified Glasgow prognostic score, PI: Prognostic index, PNI: Prognostic nutritional index, SCC-Ag: squamous cell carcinoma -related antigen, HR: hazard ratio, $\mathrm{CI}$ : confidence interval.

NLR, CRP/Alb and combined NLR+ CRP/Alb were adjusted separately in multivariable models. Results from the multivariable model which included combined NLR + $\mathrm{CRP} / \mathrm{Alb}$ score are indicated in bold. The cut-off values for CRP/Alb, NLR and PLR were determined by the method described in statistic analysis. CRP, neutrophil count and SCC-Ag were categorized according to clinical normal reference range. 

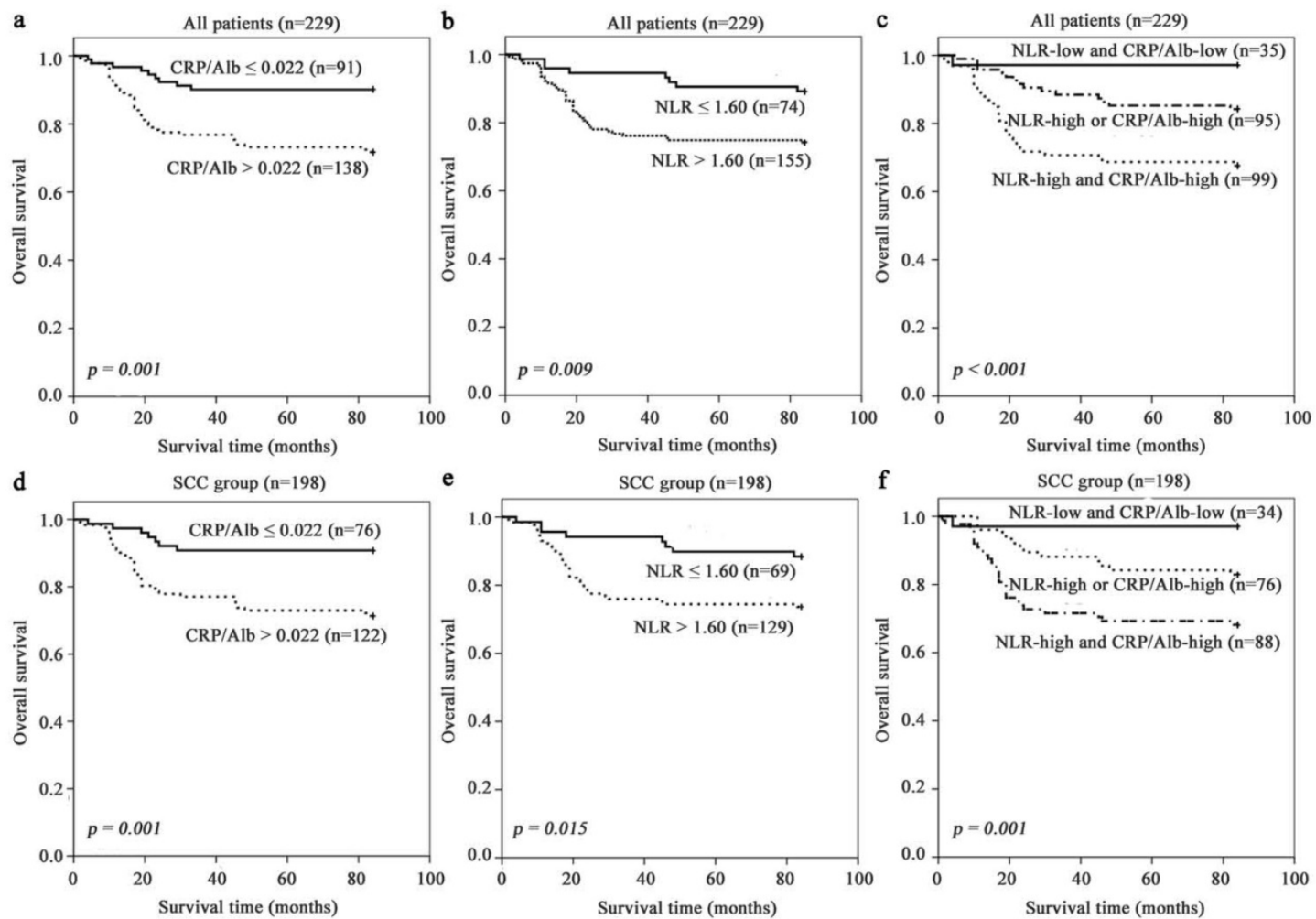

Fig. 1 Prognosis significance of NLR, CRPIAlb ratio in all cervical cancer and SCC subgroup. The 7-year OS rate was calculated by the Kaplan-Meier method and analyzed by the log-rank test. (a, d) OS based on NLR in all patients and SCC patient. (b, e) OS based on CRP/Alb ratio in all patients and SCC patient. $(c, f)$ OS of combined NLR and CRP/Alb ratio-based categorisation in all patients and SCC patient.

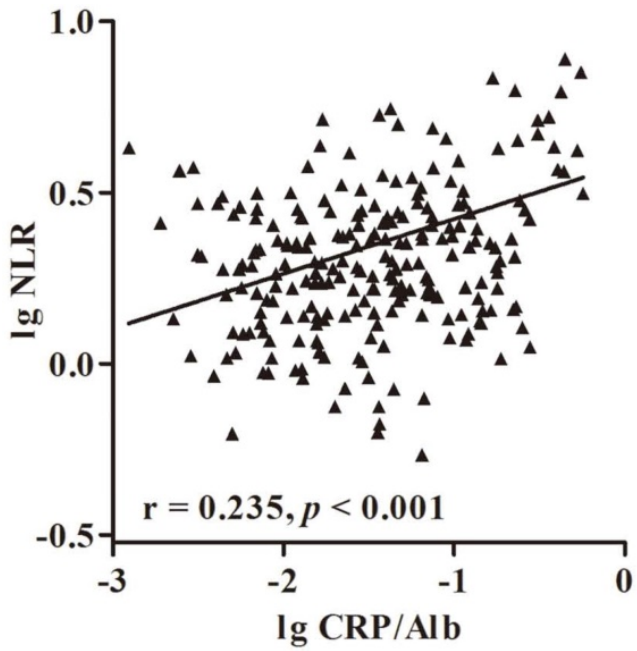

Fig. 2 Correlation of serum NLR and CRPIAlb ratio in 229 patients with cervical cancer. The Pretreatment NLR was positive correlation with CRP/Alb ratio $(r=0.235, p<0.001)$

\section{Associations between NLR and CRPIAlb Ratio with Clinicopathological Factors of Patient with SCC}

The clinicopathological characteristics of patients based on NLR and CRP/Alb ratio are shown in Table 2. NLR was statistically associated with SCC-Ag $(p=$
$0.028)$, although weakly correlated as a continuous variable (Spearman's rho coefficient 0.179; $p=0.011$ ). However, NLR was not associated with age, FIGO stages, histological grade, tumor size, and LN metastasis. An elevated CRP/Alb ratio $(p>0.022)$ was significantly associated with older patients $(p<0.001)$, more advanced FIGO stages $(p<0.001)$ and high SCC-Ag concentration $(p<0.001)$. CRP/Alb ratio also significantly correlated with age and SCC-Ag as a continuous variable (Spearman's rho coefficient 0.325 and 0.348 respectively; both $p<0.001$ ).

\section{Discussion}

In this study, we investigated the prognostic value of eight clinicopathological variables (age, FIGO stages, histological typing, histological grade, tumor size, LN metastasis treatment methods and SCC-Ag) and 10 systemic inflammatory parameters in cervical cancer and SCC subgroup. Our results showed that $\mathrm{CRP}$ and CRP/Alb ratio was a prognostic factor for OS in cervical cancer, but not an independent prognostic factor. However, it resulted to be an importantly independent prognostic factor for OS in SCC subgroup. Barbara analysed prognostic value of serum CRP in rare histological subtype adenocarcinoma of the uterine cervix. They find that 
CRP level can be seen as an additional independent prognostic parameter in patients with the rare histological subtype adenocarcinoma of the uterine cervix [11]. CRP/Alb was convenient for testing in clinical laboratories [18], and it was a sensitive, reliable convenient and a low-cost prognostic marker in cervical cancer. In addition, we found that combined NLR and CRP/Alb ratio could enhance the prognostic value in patients with cervical cancer.

Table 2. Associations of NLR and CRP/Alb ratio with clinicopathological factors of 198 patient with SCC

\begin{tabular}{|c|c|c|c|c|c|c|}
\hline \multirow[t]{2}{*}{ Characteristic } & \multicolumn{2}{|c|}{ NLR, $n$} & \multirow[t]{2}{*}{$p$ value } & \multicolumn{2}{|c|}{ CRP/Alb ratio, $n$} & \multirow[t]{2}{*}{$p$ value } \\
\hline & $\leq 1.60$ & $>1.60$ & & $\leq 0.022$ & $>0.022$ & \\
\hline Age(years) & & & 0.343 & & & $<0.001^{*}$ \\
\hline$<44$ & 31 & 49 & & 43 & 37 & \\
\hline$\geq 44$ & 38 & 80 & & 33 & 85 & \\
\hline FIGO stages & & & 0.699 & & & $<0.001^{*}$ \\
\hline I & 45 & 76 & & 62 & 59 & \\
\hline II & 21 & 45 & & 14 & 52 & \\
\hline IIIand IV & 3 & 8 & & 0 & 11 & \\
\hline Histologic grade & & & 0.741 & & & 0.892 \\
\hline G1/G2 & 32 & 63 & & 36 & 59 & \\
\hline G3 & 37 & 66 & & 40 & 63 & \\
\hline Tumor size, cm & & & 0.240 & & & 0.461 \\
\hline$<4$ & 55 & 93 & & 59 & 89 & \\
\hline$\geq 4$ & 14 & 36 & & 17 & 33 & \\
\hline LN metastasis & & & 0.551 & & & 0.275 \\
\hline No & 57 & 102 & & 64 & 95 & \\
\hline Yes & 12 & 27 & & 12 & 27 & \\
\hline Treatments & & & $0.030^{*}$ & & & $0.027^{*}$ \\
\hline Surgery alone & 19 & 20 & & 21 & 18 & \\
\hline $\begin{array}{l}\text { Surger and } \\
\text { chemotherapy } \\
\text { and(or) radiotherapy }\end{array}$ & 46 & 89 & & 50 & 85 & \\
\hline $\begin{array}{l}\text { Chemotherapy } \\
\text { and(or) radiotherapy }\end{array}$ & 4 & 20 & & 5 & 19 & \\
\hline SCC-Ag & & & $0.028^{*}$ & & & $<0.001^{*}$ \\
\hline$\leq 1.5$ & 46 & 65 & & 58 & 53 & \\
\hline$>1.5$ & 23 & 64 & & 18 & 69 & \\
\hline $\begin{array}{l}p \text { values were calculated } \\
\text { considered as statisticall }\end{array}$ & by $\mathrm{Ch}$ & $\begin{array}{l}\text { Square } \\
\text { icant. }\end{array}$ & & Fisher's & xact test, & $<0.05$ \\
\hline $\begin{array}{l}\text { Abbreviations: FIGO Int } \\
\text { lymph node, NLR the ne } \\
\text { protein/Albumin ratio, }\end{array}$ & ternati & al Fed & 舟 & necol & and Obs & rics, $L N$ \\
\hline
\end{tabular}

Low serum albumin level is correlated with malnutrition and weight loss and it is a risk factor for cancer mortality. However, our finding of a negative association between serum CRP or albumin and prognosis on multivariate analysis is the opposite to the findings of another studies [19-20]. This is due to the fact that our study included various inflammation-related factors to evaluate a prognostic value in cervical cancer, which can better represent the inflammatory conditions of patients. In general, merging CRP and albumin into a new index might have a prognostic value in inflammation, and better predict SCC patients OS. In all cervical cancer patients, $\mathrm{CRP} / \mathrm{Alb}$ ratio was not an independent prognostic factor, which might be due to the small samples number of non SCC patients. Thus, a large-scale prospective validation should be performed in non-SCC group.

mGPS was calculated with CRP and albumin (same as CRP/Alb ratio), which has been reported to be a good prognostic marker in various cancers. Although the multivariate analysis showed that mGPS was not an independent prognostic factor both in cervical cancer and SCC group, the comparison of AUC value identified no differences in the discriminatory ability between mGPS and CRP/Alb ratio (Supplementary Fig. 1). Polterauer et al also demonstrated that mGPS can be used as a survival predictor in cervical cancer [21]. What further caught our attention was that when classified by the mGPS $92.0 \%$ of patients were classified in the group of a 0 score in our study while $72.5 \%$ in Polterauer [21], suggesting that mGPS could not distinguish the survival differences of most of the patients. As a continuous variable, $\mathrm{CRP} / \mathrm{Alb}$ ratio might have the ability to identify slight differences among patients classified into the same group by the mGPS score. Therefore CRP/Alb might be a more significant inflammation-related factors than the mGPS to predict survival.

In addition, an elevated NLR was independently associated with poor OS in 229 patients with cervical cancer, even in SCC subgroup. This observation further supported the correlation between chronic inflammation and poor OS in cancer patients. Our finding of positive association between elevated NLR and poor prognosis was consistent with the results obtained from 1061 cervical cancer patients in Korea [22]. However, Zhang et al found that NLR was an independent prognostic marker for progression-free survival, but not for OS of patients with cervical cancer treated with initial radical surgery [23]. Furthermore, Wang et al found that NLR is not associated with the survival of cervical cancer patients treated with neoadjuvant chemotherapy and radical hysterectomy [24]. The possible reason was due to the treatment methods, which triggered host inflammatory response, immune response, and their imbalance. The results in our study also showed the prognostic significance of NLR according to different treatment methods by the Kaplan-Meier method and analyzed by the log-rank test (Supplementary Fig. 2).

Spearman's rank correlation analysis revealed that NLR was positively correlated with CRP/Alb ratio. The biological mechanism justifying the chronic inflammation role in cancer is yet to be elucidated. It is well known that cervical cancer not only can develop at sites of inflammation subsequent to human papillomavirus infection, but can also trigger regional inflammatory responses, releasing proinflammatory cytokines around the tumor that result in 
inflammatory microenvironment [25]. IL-6, as an important proinflammatory cytokine, stimulate the production of CRP and the production of neutrophils in the bone marrow, which is important for tumor angiogenesis [26]. Since we observed a strong correlation between NLR and CRP/Alb ratio, this phenomenon showed the complex interaction between the host immune system and the inflammatory tumor microenvironment, potentially influencing patient survival [27].

Combined NLR and CRP/Alb ratio resulted in the lowest 7-year survival ratio of the patient with CRP/Alb-high and NLR-high among the three groups. These results add further support to the fact that cervical cancer is related to systemic inflammatory response and nutritional decline, with subsequent poor outcome. These results further demonstrated the positive relationship between NLR and CRP/Alb ratio. Moreover, they highlighted the prognostic effect of combining NLR and CRP/Alb ratio. The prognostic value of combined CRP/Alb ratio and NLR should be further evaluated in a larger number of patients.

In our study, NLR and CRP/Alb ratio were associated with SCC-Ag concentration in SCC group. These results indicated that inflammation might be correlated with SCC-Ag. It has been reported that in asthma, a chronic airway inflammatory disease, both SCC-Ag 1 and SCC-Ag 2 are induced by two related Th2-type cytokines, IL-4 and IL-13, and that SCC-Ag expression is increased in bronchial asthma patients [28-29]. However, it is unclear whether the chronic inflammation, which is reflected by elevated NLR and $\mathrm{CRP} / \mathrm{Alb}$ ratio, can influence SCC-Ag expression in cervical cancer. Further studies are needed to clarify this aspect.

Besides determining a prognostic value of inflammation-related factors, we correlated NLR and $\mathrm{CRP} / \mathrm{Alb}$ ratio with clinicopathological parameters in SCC group. On the one side, we found that elevated $\mathrm{CRP} / \mathrm{Alb}$ ratio was associated with old patients and more advanced FIGO stages. The results might be explained by the following reasons: (1) Albumin was correlated with the patient's physical conditions. Malnutrition is common in old patients [30]. (2) Serum CRP levels are correlated with tumor stages in various cancers including cervical cancer. The higher the tumor stage, the greater the inflammatory response and the higher the CRP serum leve. (3) Hypoalbuminemia is suggested to predict progressive nutritional decline of patients with advanced cancer [31]. Older age and more advanced FIGO stages are both traditionally poor predictors of patients with cancer, which further support that elevated CRP/Alb ratio was associated with poor performance status.
Our study had several limitations. The results are based on single-center. Patients' number in some subgroups is small. This study was focused on Chinese population. Since cancer distribution is influenced by population, gender and geographic locations, it is problematic to associate our results to other ethnic groups.

In conclusion, our study demonstrated that $\mathrm{CRP} / \mathrm{Alb}$ ratio was a precise, convenient and inexpensive prognostic marker in cervical cancer, especially in case of SCC patients. In addition, the combination between CRP/Alb ratio and NLR could be used to better predict prognosis in patients with cervical cancer.

\section{Supplementary Material}

Supplementary figures.

http://www.jcancer.org/v09p1877s1.pdf

\section{Acknowledgements}

We thank the staff at the Director of Clinical Laboratories, Sun Yat-sen University Cancer Center for providing support on research conditions in this study.

\section{Author contribution}

H.C. and S.-L.C. proposed, designed and revised the article. X.H. and J.-P.L. designed, summarized data and revised this article. X.-H.L., J.-P.Z. and Q.-Y.Z. collected articles, summarized data, did statistical work and drafted the manuscript. All authors reviewed this manuscript and approved the final draft.

\section{Competing Interests}

The authors have declared that no competing interest exists.

\section{References}

1. Torre LA, Bray F, Siegel RL, Ferlay J, Lortettieulent J, Jemal A. Global cancer statistics, 2012. Ca A Cancer Journal for Clinicians. 2015; 65: 87.

2. Quinn MA BJ, Odicino F, Maisonneuve P, Beller U, Creasman WT, Heintz AP, Ngan HY, Pecorelli S. Carcinoma of the cervix uteri. FIGO 26th Annual Report on the Results of Treatment in Gynecological Cancer. Int J Gynaecol Obstet. 2006; Nov: 61.

3. Ferrandina G, Legge F, Fagotti A, Fanfani F, Distefano M, Morganti A, et al. Preoperative concomitant chemoradiotherapy in locally advanced cervical cancer: safety, outcome, and prognostic measures. Gynecologic Oncology. 2007; 107: S127-S32.

4. Rosa DD, Medeiros LR, Edelweiss MI, Bozzetti MC, Pohlmann PR, Stein AT, et al. Adjuvant platinum-based chemotherapy for early stage cervical cancer: John Wiley \& Sons, Ltd; 2009.

5. Petignat P, Loubeyre P. Should we modify the current FIGO staging system for early-stage cervical cancer? Expert Review of Anticancer Therapy. 2008; 8: 1015-7.

6. Brenner DE, Whitley NO, Prempree T, Villasanta U. An evaluation of the computed tomographic scanner for the staging of carcinoma of the cervix. cancer. 1982; 50: 2323-8.

7. Sturgeon CM, Duffy MJ, Hofmann BR, Lamerz R, Fritsche HA, Gaarenstroom $\mathrm{K}$, et al. National Academy of Clinical Biochemistry Laboratory Medicine Practice Guidelines for use of tumor markers in liver, bladder, cervical, and gastric cancers. Clinical Chemistry. 2010; 56: 1-48. 
8. Lee YY, Choi CH, Sung CO, Do IG, Huh S, Song T, et al. Prognostic value of pre-treatment circulating monocyte count in patients with cervical cancer: comparison with SCC-Ag level. Gynecologic Oncology. 2012; 124: 92.

9. Gadducci A, Tana R, S, Genazzani A. The serum assay of tumour markers in the prognostic evaluation, treatment monitoring and follow-up of patients with cervical cancer: a review of the literature. Critical Reviews in Oncology/hematology. 2008; 66: 10-20.

10. Reesinkpeters N, Van dVJ, Ten Hoor KA, Boezen HM, de Vries EG, Schilthuis MS, et al. Preoperative serum squamous cell carcinoma antigen levels in clinical decision making for patients with early-stage cervical cancer. Journal of clinical oncology. 2005; 23: 1455-62.

11. Bodner-Adler B, Kimberger O, Schneidinger C, Kölbl H, Bodner K. Prognostic Significance of Pre-treatment Serum C-Reactive Protein Level in Patients with Adenocarcinoma of the Uterine Cervix. Anticancer research. 2016; 36: 4691.

12. Kasymjanova G, Macdonald N, Agulnik JS, Cohen V, Pepe C, Kreisman H, et al. The predictive value of pre-treatment inflammatory markers in advanced non-small-cell lung cancer. Current Oncology. 2010; 17: 52-8.

13. Kinoshita A, Onoda H, Imai N, Iwaku A, Oishi M, Tanaka K, et al. The C-Reactive Protein/Albumin Ratio, a Novel Inflammation-Based Prognostic Score, Predicts Outcomes in Patients with Hepatocellular Carcinoma. Annals of surgical oncology. 2015; 22: 803-10.

14. Proctor M, Morrison D, Talwar D, Balmer S, O'reilly D, Foulis A, et al. An inflammation-based prognostic score (mGPS) predicts cancer survival independent of tumour site: a Glasgow Inflammation Outcome Study. British journal of cancer. 2011; 104: 726.

15. Buzby GP, Mullen JL, Matthews DC, Hobbs CL, Rosato EF. Prognostic nutritional index in gastrointestinal surgery. American Journal of Surgery. 1980; 139: 160-7.

16. Wang D, Luo H, Qiu M, Wang Z, Zhang D, Wang F, et al. Comparison of the prognostic values of various inflammation based factors in patients with pancreatic cancer. Medical Oncology. 2012; 29: 3092-100.

17. Galon J, Costes A, Sanchezcabo F, Kirilovsky A, Mlecnik B, Lagorcepagès C, et al. Type, Density, and Location of Immune Cells Within Human Colorectal Tumors Predict Clinical Outcome. Science. 2006; 313: 1960-4.

18. Póvoa P. C-reactive protein: a valuable marker of sepsis. Intensive Care Medicine. 2002; 28: 235-43.

19. Polterauer S, Grimm C, Zeillinger R, Heinze G, Tempfer C, Reinthaller A, et al. Association of C-reactive protein (CRP) gene polymorphisms, serum CRP levels and cervical cancer prognosis. Anticancer research. 2011; 31: 2259.

20. Polterauer S, Grimm C, Tempfer C, Sliutz G, Speiser P, Reinthaller A, et al. $\mathrm{C}$-reactive protein is a prognostic parameter in patients with cervical cancer. Gynecologic Oncology. 2007; 107: 114-7.

21. Polterauer S, Grimm C, Seebacher V, Rahhal J, Tempfer C, Reinthaller A, et al. The inflammation-based Glasgow Prognostic Score predicts survival in patients with cervical cancer. International Journal of Gynecological Cancer Official Journal of the International Gynecological Cancer Society. 2010; 20: $1052-7$.

22. Lee YY, Choi $\mathrm{CH}$, Kim HJ, Kim TJ, Lee JW, Lee JH, et al. Pretreatment neutrophil:lymphocyte ratio as a prognostic factor in cervical carcinoma. Anticancer research. 2012; 32: 1555.

23. Zhang Y, Wang L, Liu Y, Wang S, Shang P, Gao Y, et al. Preoperative neutrophil-lymphocyte ratio before platelet-lymphocyte ratio predicts clinical outcome in patients with cervical cancer treated with initial radical surgery. International Journal of Gynecological Cancer Official Journal of the International Gynecological Cancer Society. 2014; 24: 1319.

24. Wang D, Wu M, Feng FZ, Huang HF, Yang JX, Shen K, et al. Pretreatment neutrophil-to-lymphocyte and platelet-to-lymphocyte ratios do not predict survival in patients with cervical cancer treated with neoadjuvant chemotherapy and radical hysterectomy. Chin Med J (Engl). 2013; 126: 1464-8.

25. Deivendran S, Marzook KH, Pillai MR. The role of inflammation in cervical cancer. Inflammation and Cancer: Springer; 2014. p. 377-99.

26. Kusumanto YH, Dam WA, Hospers GA, Meijer C, Mulder NH. Platelets and granulocytes, in particular the neutrophils, form important compartments for circulating vascular endothelial growth factor. Angiogenesis. 2003; 6: 283.

27. Mcmillan DC. Systemic inflammation, nutritional status and survival in patients with cancer. Current Opinion in Clinical Nutrition \& Metabolic Care. 2009; 12: 223.

28. Sakata Y, Arima K, Takai T, Sakurai W, Masumoto K, Yuyama N, et al. The squamous cell carcinoma antigen 2 inhibits the cysteine proteinase activity of a major mite allergen, Der p 1. Journal of Biological Chemistry. 2004; 279: 5081.

29. Izuhara K. The role of interleukin-4 and interleukin-13 in the non-immunologic aspects of asthma pathogenesis. Clinical Chemistry \& Laboratory Medicine Cclm. 2003; 41: 860.

30. Viera K, Jaroslav R, Maria G, Adrian K. Ten-year all-cause mortality in hospitalized non-surgical patients based on nutritional status screening. Public Health Nutrition. 2015; 18: 2609-14.

31. Dewys WD, Begg C, Lavin PT, Band PR, Bennett JM, Bertino JR, et al. Prognostic effect of weight loss prior to chemotherapy in cancer patients. Eastern Cooperative Oncology Group. American Journal of Medicine. 1980; 69: 491 . 